\title{
La estructura de sucesión en el libro III de los Fastos: de Rómulo a Numa como prototipo de Augusto*
}

\author{
Pablo Martínez Astorino \\ Conicet/Universidad Nacional de La Plata \\ pmastorino@gmail.com \\ ORCID iD: http://orcid.org/0000-0002-3169-8021
}

\section{The succession-structure in Fasti III: from Romulus to Numa as a prototype of Augustus}

En los Fastos la imagen de Rómulo resulta insuficiente para aludir a Augusto porque el Augusto contemporáneo de Ovidio ya no obedece al prototipo romúleo de nuevo fundador de la ciudad y se asemeja, en cambio, a Numa, rey consagrado a la paz, a los ritos y al conocimiento de los astros. Esta circunstancia se dramatiza especialmente en II 119144 y en I 27-44 y III 73-166. No obstante, el libro III es el lugar de la obra en el que más se enfatiza esa dramatización, ya que poco menos de la mitad del libro (la primera mitad) está dedicada sucesivamente a Rómulo y a Numa. En este trabajo se estudia cómo influye la estructura de sucesión en la construcción del personaje de Augusto, con conclusiones sobre la construcción poética ovidiana de la historia: Ovidio
In the Fasti the image of Romulus fails to allude to Augustus because Ovid's contemporary Augustus no longer responds to the Romulean prototype of a new founder of the city, and resembles Numa instead, king devoted to peace, rites and to the study of the stars. This circumstance is especially dramatized in II 119-144 as well as in I 27-44 and III 73-166. Nevertheless, Book III is the locus of the work in which this dramatization is most emphasized, since little less than a half of the Book (the first half) is devoted successively to Romulus and to Numa. In this paper we study how this succession-structure influences the construction of Augustus as a character, with conclusions about the Ovidian poetic construction of history: Ovid

* Este trabajo, versiones previas del cual fueron presentadas como ponencias y finalmente como conferencia en las "IV Jornadas Nacionales del Centro de Literaturas y Literaturas Comparadas" (FaHCE-UNLP, 4. 11. 2016), se inserta en mi investigación como Investigador Adjunto del Conicet "La construcción poética de la historia romana en la poesía augustea: Virgilio y Ovidio". Por consejo del evaluador anónimo, he ampliado las conclusiones (me ha parecido más adecuado insertar en ese sector, antes que en el inicio, la explicación sobre las perspectivas teóricas acerca de la historia que he sostenido en otros artículos). Agradezco esta y otras observaciones que indudablemente han dotado de una mayor claridad a mi argumentación.

Copyright: (C) 2018 CSIC. Este es un artículo de acceso abierto distribuido bajo los términos de la licencia de uso y distribución Creative Commons Reconocimiento 4.0 Internacional (CC BY 4.0). 
presenta $s u$ interpretación del Augusto posterior a Accio, que resulta más bien un artificio poético.

Palabras clave: Ovidio; Fastos; Rómulo; Numa; Augusto; sucesión. presents his interpretation of the after-Actium $\mathrm{Au}-$ gustus, which becomes rather a poetic device.

Key words: Ovid; Fasti; Romulus; Numa; Augustus; succession.

Cómo citar este artículo / Citation: Martínez Astorino, Pablo 2018: «La estructura de sucesión en el libro III de los Fastos: de Rómulo a Numa como prototipo de Augusto», Emerita 86 (1), pp. 133-149.

\section{Introducción: status quaestionis e hipótesis}

La asociación de Augusto con Rómulo fue propiciada por Augusto mismo. Suetonio (Aug. 7.2; 95) y Dión Casio (XLVI 46.23; LIII 16.7) refieren una toma de auspicios durante su primer consulado que lo equipara a Rómulo (se le aparecieron doce buitres) y recuerdan que Augusto, en tanto restaurador de Roma, prefería el nombre de Rómulo al que finalmente recibión ${ }^{1}$. Si descartó ese nombre, fue por sus asociaciones con la tiranía ${ }^{2}$. No obstante, en el Foro de Augusto, dedicado en el 2 a. C., podía apreciarse su imagen junto con la de Eneas, Julio César y el propio Augusto. En cuanto a Numa, quien también tenía un destacado lugar en dicho foro, hay una alusión que, en el período inmediatamente anterior y contemporáneo de Ovidio, se convirtió en un tópico: el motivo de la pax. Tito Livio (I 19.1-3) y Virgilio (Aen. I 293 ss.) recuerdan que Augusto cerró las puertas del templo de Jano ${ }^{3}$. El primero incluye este recuerdo en el comienzo de la historia de Numa, pero, aun sin esa relación directa, el acto de Augusto remite a la tradición de ese rey que, al cerrar el templo de Jano recién inaugurado al pie del Argileto, aparece en la historia romana como el primer pacificador ${ }^{4}$.

${ }^{1}$ Cf. Hinds 1992, p. 127 s.

2 Como observa Urban 2005, p. 91, que cita a Alföldi 1973, p. 13: «Da der Name Romulus jedoch Assoziationen mit Königtum und Tyrannis aufkommen liess, und Caesar, dem man Königsambitionen nachgesagt hatte, eine besondere Vorliebe für diesen Namen gezeigt hatte, suchte und fand Augustus einen Namen, der an die Stadtgründung Roms erinnerte, ohne die mit dem Namen Romulus verbundenen negativen Konnotationen wachzurufen, und der ihm zudem eine 'sakralangehauchte Autorität verlieh'».

3 Asimismo, el propio Augusto, R.G. 13 (seguimos el texto de Brunt y Moore, 1973²): Ianum Quirinum, quem claussum esse maiores nostri uoluerunt cum per totum imperium populi Romani terra marique parta uictoriis pax, cum, priusquam nascerer, a condita urbe bis omnino clausum fuisse prodatur memoriae, ter me principe senatus claudendum esse censuit.

${ }^{4}$ Austin 1971, ad Aen. I 293 ss. La relación se hace aún más estrecha si es cierta la propuesta de Ogilvie 1965, p. 93 s. de que quizás el mismo Augusto haya resucitado una 
La crítica sobre los Fastos (y también la mera lectura atenta) ve en Rómulo y Numa dos prototipos de Augusto. En las últimas dos décadas esta asociación ha tenido, no obstante, aproximaciones e interpretaciones dispares. Están: 1. los que opinan que la ambigüedad en torno a la figura de Rómulo no concierne a su deificación y a su significación augustea 5 ; 2 . los que consideran que toda la representación de Rómulo es crítica o irónica y que esta circunstancia, por ende, afecta a la representación de Augusto ${ }^{6}$; 3. los que creen que en la obra hay cierta crítica al prototipo romúleo y que (o, también, porque) Ovidio pretende asociar a Augusto con Numa, un rey consagrado a la paz y al conocimiento de los astros, e incluso con el propio poeta ${ }^{7}$; 4 . los

tradición caída en desuso (cf. Hinds 1992, p. 130): «The tradition that they were closed in 235 (or $241 \ldots$...) after the First Punic War derives at least from the historian Piso (ap. Varro, de Ling. Lat. 5. 165) and there may well have been an authentic notice of it. It is, however, surprising that there should be no other recorded instance of the doors being shut, for even in the centuries after 241 there were numerous periods of total peace. This may suggest that the practice of closing the doors as a symbol of peace was not in fact generally recognized but was resuscitated either by antiquarians in the closing years of the Republic or by Octavian himself as a propaganda gesture. Some such period of desuetude would also account for the diversity of legends about the founder of the shrine». Por su parte, Deremetz 2013, p. 236, retomando a Svenbro 1988, recuerda que la Vida de Numa de Plutarco parece ser un mito etimológico compuesto o inventado por un mitógrafo griego «... to illustrate one of the conceptions that the Greeks had of the problematic relationships between law and writing. Numa is, for them, the incarnation of nomos empsuchos when he is alive and, after his death, of the nomos apsukhos, whereas Lycurgus for his part is the incarnation of the nomos basileus». Para la consideración de Numa como modelo de Augusto, cf. Galinsky 1996, pp. 84, 282, 346; para la relación Numa-Augusto en las monedas, cf. Galinsky 1996, pp. 34-37 y, más recientemente, Györi, 2013, pp. 89-108, que ofrece una detallada comparación de Augusto y Numa (97-100) y concluye que los asses de Augusto/Numa del 23 a. C. sobre los que trata en la primera parte de su artículo, «... are the contemporary numismatic parallel to Virgil's heroic parade» (100) del libro VI de la Eneida, compuesto en el 23 a. C. bajo la atmósfera de los Ludi Saeculares, que habrían sido planeados para ese año y luego postergados. En ese desfile, Augusto se ubicaría entre Rómulo y Numa como un rey más, para asociarse con este último (cf. n. 77 del trabajo de Györi y n. 26 del presente trabajo), con quien, como hijo adoptivo de Julio César, estaba emparentado (v. Györi 2013, p. 89; Suet., Iul. 6). Györi 2013, p. 89 recuerda: «He (Numa) appears in Virgil's 'parade of heroes' in Book 6 of the Aeneid as he later does in Augustus' 'hall of fame' in the Forum Augustum».

${ }^{5}$ Salzman 1998, pp. 327, 329; Herbert-Brown 1994, pp. 49-51.

${ }^{6}$ Hinds 1992.

7 Stok 1992, pp. 47-73. Monella 2008, pp. 85-107. Con respecto a la asociación sólo con el poeta, Pasco-Pranger 2002, pp. 291-311. Garani 2014 estudia la filiación empedoclea 
que opinan que, aun asociando a Augusto con Numa, la posición del poeta podría ser ambigua ${ }^{8}$.

Nuestra visión sobre estos personajes se acerca a la de los críticos alineados en los puntos 1 y 3. Creemos que en los Fastos la imagen de Rómulo resulta insuficiente para aludir a Augusto porque el Augusto contemporáneo del Ovidio poeta ya no obedece al prototipo romúleo de nuevo fundador de la ciudad y se asemeja, en cambio, a Numa, rey consagrado a la paz, a los ritos y al conocimiento de los astros. Esta circunstancia se dramatiza especialmente en la sýnkrisis entre Rómulo y Augusto (II 119-144) y en la sýnkrisis entre Rómulo y Numa a propósito del calendario (I 27-44, III 73-166), pasajes en los que la figura de Rómulo aparece censurada. No obstante, el libro III es el lugar de la obra en el que más se enfatiza esa dramatización, ya que poco menos de la mitad del libro (la primera mitad) está dedicada sucesivamente a Rómulo y a Numa. En este trabajo estudiaremos cómo influye la estructura de sucesión, i.e. el pasaje explícito (en términos de representación) de un prototipo o imagen a otro, en la construcción del personaje de Augusto, y analizaremos brevemente en sus conclusiones si, en términos de construcción poética de la historia reciente (según venimos estudiando en trabajos anteriores), se trata de una auténtica interpretación de la historia o de un artificio poético.

\section{El libro III: de Rómulo a Numa}

El libro III de los Fastos trata sobre el primer mes del calendario romúleo y el tercero a partir de la reforma de Numa, i. e. marzo, mes que toma su nombre del dios Marte. El comienzo del libro pone en evidencia la sorpresa del poeta ante su obligación en términos compositivos, que era escribir sobre el mes de la guerra en un libro sobre el calendario, las causas y las constelaciones (Fast. I 1-2). La hipotética pregunta de Marte obtiene, por ese motivo, una respuesta resignada y algo irónica del poeta:

y lucreciana de la imagen de poeta / uates de Numa, dotada por la filosofía de una profunda significación política: «All in all, Numa is revealed to be standing in as both the poet and the emperor» (p. 160).

${ }^{8}$ Littlewood 2002, pp. 175-197. Galinsky 2013, p. 33 s., esp. 34, n. 24, opina que, al igual que en el caso de César, Augusto se asocia a Rómulo tomando distancia de sus «liabilities». 
forsitan ipse roges quid sit cum Marte poetae:

a te qui canitur nomina mensis habet. (Fast. III 3-4) ${ }^{9}$

Quizás tú mismo te preguntes qué tienen en común el poeta y Marte:

de ti recibe el nombre el mes que cantamos.

Nada más parece vincularlos. Ese rechazo obedece desde el punto de vista poético a una recusatio que ha sido explicitada al inicio del libro I con el magistral hexámetro Caesaris arma canant alii: nos Caesaris aras, I 13 («que otros canten las armas de César; nosotros cantaremos sus altares») ${ }^{10}$. La historia que Ovidio elegirá referir a continuación es una historia en la que el dios no tuvo necesidad de armas: sus amores con la vestal Rea Silvia. El consejo del poeta de imitar a Minerva y deponer por un tiempo las armas adquiere el tono de la ironía cómplice: inuenies et quod inermis agas, III 8 ( «sin armas también encontrarás qué hacer»). En el incipit del verso que da comienzo a la historia, por la repetición del término inermis, se sugiere que el dios de la guerra es, además de un dios desarmado, un dios inofensivo (sentido derivado del término), presa del amor de una mujer: tum quoque inermis eras, cum te Romana sacerdos/ cepit..., III 9-10 («también entonces estabas sin armas/ eras inofensivo, cuando te tomó la romana sacerdotisa...») $)^{11}$.

Sin embargo, la historia que se contará no será sólo una historia amorosa (o al menos una más), circunstancia que se aclara en el verso 10: Rea Silvia «tomó» al dios para que éste «diera a la Ciudad grandes semillas», i. e. Rómulo y Remo. La posición activa de la vestal se asignará más tarde al dios, quien, como se lee en los versos 20-21, no se mostrará inofensivo: la ve, la desea y, sobre todo, la posee (potitur -20). El relato se concentra luego en el discurso de la vestal y en las circunstancias del nacimiento y la crianza de los gemelos hasta llegar a su juventud. En este punto, algunas de las caracterís-

9 Todas las citas de Fastos corresponden a la edición de Alton-Wormell-Courtney 2005. Las traducciones castellanas nos pertenecen.

10 Cf. Barchiesi 1994, p. 164 s. Stok 1992, p. 69 declara que el tema del proemio es retomado en los proemios paralelos de los libros II, III y IV, lo que enfatiza su propósito de que los Fastos sean una alternativa a la Eneida (p. 72): «L'intero poema, del resto, con i suoi previsti dodici libri, voleva essere, più ancora delle Metamorfosi, l'alternativa all'epica, e quindi all'Eneide. Questo carattere di alternativa all'Eneide, che è solo sancito dal rovesciamento del luogo politicamente più impegnato del poema virgiliano, chiarisce, direi, il senso delle dichiarazioni proemiali di Ovidio, e l'aspirazione ad un poema capace di cantare i maiora senza essere epico».

11 Gaffiot, s. u. 2 fig. a: 'inoffensif'; $O L D$, s. u. 1c. 
ticas que se les asignan a los gemelos, y en especial a Rómulo, no pueden dejar de leerse, conforme al paralelo que explicitamos al comienzo, en relación con Augusto. Aunque sensibles a las leyes, los gemelos son propensos a las armas, por medio de las cuales hacen justicia frente a delincuentes y usurpadores:

omnibus agricolis armentorumque magistris

Iliadae fratres iura petita dabant.

saepe domum ueniunt praedonum sanguine laeti

et redigunt actos in sua rura boues.

...

Romuleoque cadit traiectus Amulius ense, regnaque longaeuo restituuntur auo. (III 61 ss.)

Los hermanos hijos de Ilia dictaban las leyes que se les pedían

a todos los agricultores y a los pastores del ganado.

A menudo llegan a casa contentos por haber derramado sangre de salteadores y devuelven a sus campos los bueyes robados

$\cdots$

Y Amulio cae atravesado por la espada de Rómulo y restituyen el reino a su anciano abuelo.

La justicia se aplica asimismo a quien pueda constituir una amenaza al poder establecido, como el propio Remo. A diferencia de otras versiones de esa historia que concluye en el asesinato de Remo, se omite todo comentario ritual:

moenia conduntur, quae, quamuis parua fuerunt, non tamen expediit transiluisse Remo. (III 69-70)

Se fundan unas murallas que, aunque fueron pequeñas, sin embargo no le habría convenido a Remo haberlas traspasado.

Si bien es la historia de Rómulo, por la conocida asociación con Augusto el lector podía (y puede, en efecto) vincular el rol de los gemelos y, posteriormente, el de Rómulo con el Augusto pacificador de la Guerra Civil; en el segundo ejemplo, Remo mismo, alusivamente, podría leerse como una anticipación de la amenaza al orden, según el prisma de la propaganda augustea. Lo que, sin embargo, atenúa un poco las bondades de la asociación es cierta rudeza en la caracterización del personaje de Rómulo, que se pone de relieve de manera especial en el episodio del rapto de las Sabinas, donde 
el dios Marte apostrofa: ... patriamque dedi tibi, Romule, mentem./ «tolle preces», dixi «quod petis arma dabunt.», III 197-198 («... te di, Rómulo, el carácter de tu padre. 'Haz a un lado las plegarias', le dije, 'las armas te darán lo que pides'») ${ }^{12}$. El dios está representado algo cómicamente; además la historia del rapto se cuenta a propósito de los Matronalia y en el final se exalta la pacificación, pero se percibe cierta reserva por parte del poeta ante la imagen de Rómulo, que se asocia ineluctablemente a la violencia de Marte. Esta circunstancia, si decidimos leer el pasaje en clave augustea, repercutiría desfavorablemente en la interpretación del personaje de Augusto.

En el pasaje sobre la ordenación del calendario, que continúa un motivo ya tratado en el libro I, a propósito del mes de enero, el poeta, con un dejo de burla, nos muestra a un Rómulo pasional, que, celoso de los lazos de sangre, de cuya seguridad no permite que se dude, decide homenajear a su padre, no menos pasional que él, sin advertir que este homenaje conlleva un error astronómico, un calendario al que le faltan dos meses (III 73-78):

'arbiter armorum, de cuius sanguine natus credor et, ut credar, pignora multa dabo, a te principium Romano dicimus anno:

primus de patrio nomine mensis erit.' uox rata fit, patrioque uocat de nomine mensem:

dicitur haec pietas grata fuisse deo.

'Árbitro de las armas, de cuya sangre se cree que he nacido y, para que se crea, daré muchas garantías, a partir de ti llamamos al comienzo del año romano.

El primer mes tendrá el nombre de mi padre'.

Se cumple lo dicho y llama al mes conforme al nombre de su padre.

Cuentan que esta muestra de piedad agradó al dios.

Se insiste otra vez en la violencia (arbiter armorum) y en la rudeza, no sólo de este Rómulo de quien Marte es padre y cómplice (cf. el desafío del verso 74 y el agrado del dios, en el 78), sino de aquellos tiempos primitivos de Roma, ignorantes de la oratoria y de la astronomía. En un pasaje que puede ser leído como un intertexto y, sobre todo, como una respuesta al famoso

12 Si bien Bömer 1958, ad Fast. III 198 afirma «wenn überhaupt jemand, dann ist hier Mars der Urheber des 'Umrechts', nicht Romulus», Rómulo queda asociado a Marte. 
pasaje de las artes Romanae que Virgilio incluyó en el discurso de Anquises de la Eneida (VI 847 ss.), el poeta escribe (III 101 ss.) ${ }^{13}$ :

nondum tradiderat uictas uictoribus artes

Graecia, facundum sed male forte genus:

qui bene pugnabat, Romanam nouerat artem;

mittere qui poterat pila, disertus erat.

libera currebant et inobseruata per annum

sidera; constabat sed tamen esse deos.

non illi caelo labentia signa tenebant,

sed sua, quae magnum perdere crimen erat.

Grecia todavía no había legado las artes vencidas

a los vencedores; era un pueblo elocuente, pero poco valiente.

Quien luchaba bien conocía el arte romano;

quien podía arrojar dardos era elocuente...

Los astros corrían libres y sin ser observados

a lo largo del año, pero les constaba, sin embargo, que eran dioses.

No comprendían los signos (astros) que se deslizan en el cielo, sino los suyos (estandartes), que era un gran delito perder.

En los versos que siguen el poeta mostrará respeto por la austeridad de esos primitivos habitantes, que honraban unos estandartes rústicos de heno; pero los versos que hemos citado reflejan una cierta censura, que se evidencia especialmente en el juego de palabras con signa y en la caracterización del rústico método de elocuencia romana, aunque probablemente también en su observación de que reconocían el carácter divino de los astros, sin una consecuente preocupación por su valor científico ${ }^{14}$.

13 V. Stok 1992, p. 63 s., que cita además los pasajes asociados de Ars I 459-462 y Met. XIII 288 ss.: «La rilettura di 3, 101 ss. alla luce delle citazioni di Aen. 6, 847-53 nell' Ars e nelle Metamorfosi, non lascia dubbi, direi, sul senso dell'operazione proposta da Ovidio nei confronti del luogo virgiliano: quello di una netta presa di distanza dal giudizio formulato nell'Eneide, che è esplicitata nei termini di una difesa d'ufficio delle artes subordinate nell' Eneide all'attività militare, e che è anche, ovviamente, una presa di distanza da quella rivalutazione augustea dei valori tradizionali che costituisce, in ultima analisi, il contesto ideologico e culturale del giudizio virgiliano». Obviamente hay también una alusión a Horacio, Ep. II 1.156-157: Graecia capta ferum uictorem cepit et artes/ intulit agresti Latio.

${ }^{14}$ Stok 1992, p. 65: «Parodico, ancora, nei confronti del luogo virgiliano, ma più in generale del favore accordato dalla propaganda augustea alla simbologia militare, appare il gioco di 
La misma estructura de sucesión que se da a comienzo del libro I se repite aquí. En ese libro, el poeta, exclama (Fast. I 29-30):

scilicet arma magis quam sidera, Romule, noras, curaque finitimos uincere maior erat.

Sin duda conocías más las armas que las estrellas, Rómulo, y era mayor tu preocupación por vencer a los pueblos vecinos.

No obstante, luego de estos versos excusa a Rómulo (vv. 31 ss.), explicando que fenómenos de la naturaleza (la duración del embarazo) o acontecimientos de la realidad (la duración del luto), justificaban la creencia en que el año duraba diez meses en lugar de doce ${ }^{15}$. Esta justificación se encuentra considerablemente ampliada en el libro III, lo que sugiere un matiz en la construcción de una imagen crítica de Rómulo, y, al igual que en el I, desemboca en Numa (III 151 ss.). Sea instruido por Pitágoras -es decir, por la Graecia uicta- o amonestado por su esposa, la ninfa Egeria, Numa menses sensit abesse duos (152), «advirtió que faltaban dos meses». Lo novedoso de este pasaje, a diferencia de su correlato del libro I, es la inmediata asociación con Julio César, caracterizado como ille deus tantaeque propaginis auctor, 157 («aquel dios y autor de una progenie tan grande -i. e. Augusto»):

sed tamen errabant etiam nunc tempora, donec

Caesaris in multis haec quoque cura fuit.

non haec ille deus tantaeque propaginis auctor

credidit officiis esse minora suis,

promissumque sibi uoluit praenoscere caelum

nec deus ignotas hospes inire domos. (Fast. III 155-160)

Sin embargo, aun ahora el cálculo del tiempo era erróneo, hasta que entre muchas también ésta fue preocupación de César.

No creyó aquel dios y autor de una progenie tan grande

parole di v. 113-14, dove i signa sono contemporaneamente le costellazioni, ignote ai ueteres Romani, e le insegne militari ...; ma più in generale l'intera immagine della Roma romulea appare lontana dall'idealizzazione e dall'uso che la propaganda augustea faceva della storia più antica di Roma». Sin embargo, sugiere no hacer una lectura antiaugustea de la parodia, que sería, dice, ingenua. V. asimismo Labate 2003, p. 95 s.

${ }^{15}$ Green 2004, ad Fast. I 29 comenta: «The tone of scilicet can vary from sarcastic/ironic to simple matter-of-fact ... Ovid may be including in a spot of light-hearted banter here at Romulus' expense, but he may equally be commenting on the primary need of early Rome to develop herself militarily». 
que estas cuestiones fueran menores que sus deberes y quiso conocer de antemano el cielo que le era prometido y no entrar como un dios extranjero en una casa desconocida.

La representación del poeta afirma que el paradigma de Rómulo ha sido superado por el paradigma de Numa, quien se vincula, a través del interés de César en la astronomía, con Augusto.

Permítasenos retomar lo dicho hasta el momento y agregar una idea para completar este apartado. En los dos libros anteriores Rómulo es considerado separadamente en relación con Numa o en relación con Augusto, a través del artificio de la sýnkrisis. Hemos comentado la comparación con Numa a propósito del calendario, que, con ciertas variaciones y amplificaciones -pero, sobre todo, con el agregado esencial de la referencia indirecta a Augusto-, se repite en el libro III. La sýnkrisis entre Rómulo y Augusto ocurre en el libro $\mathrm{II}^{16}$. Si bien no se ahorran diferencias, son llamativas éstas que señalamos de manera especial (II 141-143):

uis tibi grata fuit, florent sub Caesare leges;

tu domini nomen, principis ille tenet;

te Remus incusat, ueniam dedit hostibus ille.

A ti te es agradable la fuerza; bajo César florecen las leyes.

Tú tienes el nombre de señor; aquél, el de primer ciudadano.

A ti te acusa Remo; el perdonó a sus enemigos.

Este reinado caracterizado por las leyes y la clemencia se parece, en la tradición mítico-histórica romana al de Numa. Podría decirse que Rómulo, en los Fastos, es vencido en las sýnkriseis particulares con Numa y con Augusto y que en esta última algunas características de Augusto lo acercan a Numa. Ahora bien, en el

16 V. Barchiesi 1994, p. 71, Barchiesi 1997, p. 81, quien observa que, en la comparación de Augusto con Rómulo en ocasión de celebrar el aniversario del título conferido a Augusto (2 a. C.) de pater patriae, se destruye la figura de Rómulo. V. además la interesante interpretación de Galinsky, 2013, pp. 34 s., que comenta críticamente a Barchiesi: «These comments hit the mark, but there is even more to the passage (...). Romulus was an essential figure in Augustan mythology and discourse even beyond the aspect of pater patriae. A subtext in the Ovidian passage is Augustus' rejection, around $28 \mathrm{BC}$, of being called the second Romulus. Ovid's list of Romulus' negatives mimics the arguments at the time -surely, Octavian, you don't want to be known for those qualities. But there is more of a bite: except for not taking the name, Augustus did not separate himself much from Romulus. As so often, he was having it both ways and that, I think, is one of Ovid's targets here». 
libro III la comparación, la sýnkrisis entre Rómulo y Numa adquiere, por la estructura del libro, las características de una sucesión y, por la relación intratextual de Augusto con Numa en estructura de sýnkrisis (libro II), se entiende que, en esta sucesión de Rómulo a Numa, se está aludiendo de manera textual a la sýnkrisis de Rómulo y Augusto (no se trata, pues, de una cuestión meramente cultural, como señalamos al principio). Para otorgar más énfasis, la referencia a Numa culmina, como hemos visto, en una referencia a César y a Augusto. En consecuencia, Rómulo, en el libro III, aparece desdibujado y caracterizado con rasgos aun humorísticos, porque el poeta propone en este libro de manera programática la sucesión, en relación con el princeps, de un modelo mítico-histórico a otro. No obstante, la conciencia de que el personaje de Rómulo era un prototipo de Augusto -y a la vez la propia estructura de sucesión- induce al poeta a matizar sus errores, luego de burlarse de ellos, y a que en la última historia contada en relación con Rómulo y con Marte, la referencia al rapto de las Sabinas a propósito de la fiesta de los Matronalia, se ponga el acento en la paz y el propio Marte aparezca como un dios de paz asociado a las madres (III 251-252):

mater amat nuptas: matris me turba frequentat.

haec nos praecipue tam pia causa decet.

Mi madre ama a las casadas: la multitud de mi madre, pues, me frecuenta.

Esta causa tan piadosa me conviene especialmente.

Es lícito que se mantenga la asociación entre Rómulo y Augusto en la obra, pero hay que considerar que, cuando se advierten críticas o reservas a Rómulo, es precisamente porque aparece en una estructura comparativa que conduce a su dramatización en el libro III, en una estructura de sucesión: la plenitud de Rómulo-Augusto se halla en Numa-Augusto. Hay, por otro lado, asociaciones entre Rómulo y Augusto que no pueden ser compartidas por Numa, como la apoteosis: en ese caso, no se observan críticas, precisamente porque la apoteosis posee valor cesáreo-augusteo ${ }^{17}$. Por último, está el motivo del asesinato de Remo, que aparece censurado en la sýnkrisis entre Rómulo y

17 Salzman 1998, pp. 327, 329; Herbert-Brown 1994, pp. 49-51. Gosling 2002, pp. 51-69 ofrece argumentos a favor de la tesis de que, aunque tal vez no con seguridad en consonancia con valores augusteos sino manifestando su propia posición poética, tanto en Metamorfosis (v. 806 -que incluye el posita cum casside Mauors-; vv. 823-824), como en Fastos (I 37-38; II 491-492; II 508) la representación de la apoteosis de Rómulo enfatiza el motivo de la paz y la ley, con ecos de Aen. I 292 s. y Aen. VI 851-853) -Hardie 2015, ad Met. XIV 805 s.: «Ovidio sottolinea il suo ruolo civile». 
Augusto y en cierta manera justificado en los relatos del libro IV (fundación de la ciudad) y V (los Lemuria). El tema es bastante amplio y merece un estudio separado ${ }^{18}$. Baste decir que Ovidio lo ha representado en términos menos críticos y pesimistas que, por ejemplo, Horacio en el Epodo 7.

En esta estructura sucesoria, elaborada cuidadosamente en un libro sobre el mes de marzo, asociado a Marte, el primer pasaje dedicado a Numa insiste en el motivo de la paz y en la gradual civilización del pueblo otrora rústico y belicoso (III 277-282):

principio nimium promptos ad bella Quirites molliri placuit iure deumque metu. inde datae leges, ne firmior omnia posset, coeptaque sunt pure tradita sacra coli.

exuitur feritas, armisque potentius aequum est, et cum ciue pudet conseruisse manus.

Al principio agradó - a Numa- que los quirites excesivamente dispuestos a la guerra fueran ablandados por la ley y el temor de los dioses.

Luego se dictaron leyes para que el más fuerte no lo pudiera todo y se comenzaron a honrar con pureza los ritos tradicionales.

Se aparta la ferocidad y lo justo es más poderoso que las armas y causa vergüenza irse a las manos con un ciudadano.

La primera hazaña de Numa, conforme a esta nueva orientación, no se deberá a la fuerza, sino a la inteligencia: consiste en la propiciación del rayo, obtenida luego de un duelo verbal con Júpiter.

\section{Conclusiones: representación de la historia; el caso de los Fastos}

Aunque, como se ha observado recientemente, representar historia excede los cánones de género y se define como una cuestión de tema ${ }^{19}$, la poesía, al tratar la historia, ejerce una auténtica transformación. Esto puede deducirse

18 Remitimos al libro de Wiseman 1995, esp. pp. 9-17, 103-128 y 144-150.

19 Gowing 2005, p. 11: «In the Roman conception historia was a generalizing term that applied no merely to 'historiography' or 'historical writing' - what we might today consider to be the most legitimate medium of historical inquiry - but to any attempt to transmit the past. For the Roman historia is less a genre than a definition of subject matter». Wiseman 2002, p. 362: «Even in the sophisticated Rome of the first century B.C., for many readers the distinction between proper pursuits of poets and historians was far from clear-cut, and certainly not a simple matter of literary genre». 
de la propia diferenciación entre historia y poesía que hace Aristóteles (Poet. 1451b): la poesía, a diferencia de la historia, que considera lo sucedido, se aboca a lo que puede suceder; por ende, la historia incluida en textos poéticos va a estar impregnada de artificio, de invención, de construcción ${ }^{20}$. Como lo hemos formulado en otro trabajo,

Esta circunstancia se hace posible por una distinción que, pese a lo que opinan Gowing y Wiseman, se plantea en términos de género, aunque sirve para iluminar el estatus especial de la poesía que representa historia frente a una equívoca equiparación de la labor del poeta y del historiador con la historia, y no, por cierto, para desestimar la representación poética de esa materia. En efecto, para Aristóteles (Poet. 1451b), la historia se diferencia de la poesía porque, mientras la prime-

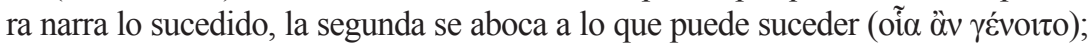
asimismo, la poesía, más filosófica y universal que la historia, se rige por el crite-

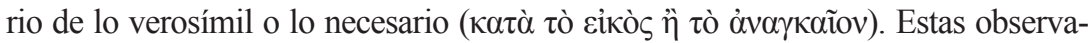
ciones de Aristóteles nos permiten inferir que la poesía, cuando representa historia, pone el acento en una labor que prioriza la transformación, la construcción, de un modo que no puede encontrarse en la historia como forma dominante ${ }^{21}$.

En lo que respecta a la poesía augustea, estudios que tienen ya cuatro décadas ${ }^{22}$ han demostrado que la etiqueta de régimen augusteo o de una ideología impuesta del principado no es del todo adecuada y que los poetas gozaron de libertad. La idea de construcción poética sugiere que todo poeta augusteo ha sido capaz de construir una representación autónoma y más o menos diferenciada de las expectativas que la política depositaba en los escritores, atendiendo al modo como

${ }^{20}$ No nos parece que constituya una auténtica objeción lo que Aristóteles afirma más abajo, que ha sido comentado por Ricoeur 1996, p. 915: «En la misma página que opone la poesía a la historia, los trágicos son alabados por haberse limitado 'a los nombres de hombres realmente atestiguados'. Y ésta es la razón: que lo posible es persuasivo: lo que no ha ocurrido, no creemos aún que sea posible; mientras que lo que ha ocurrido, es evidente que es posible' (1451b 15-18). Aristóteles sugiere aquí que, para ser persuasivo, lo probable debe tener una relación de verosimilitud con el haber-sido».

${ }^{21}$ Martínez Astorino 2014, p. 349 s. Coincidimos con Lorenzo 2011, pp. 19-21 en que la retórica, en época imperial, hizo que épica e historia se acercaran: baste considerar la representación de batallas, discursos o arengas; no obstante, la diferencia entre la historia y la poesía (o la épica en particular) se cifra en que en la primera la construcción, operada o no por la retórica, no atañe al todo sino a las partes, mientras que en la segunda toda la representación, esencialmente, queda informada por una transformación mítica.

${ }_{22}$ Galinsky 1975, pp. 210-217, 251-261; Galinsky 1996, pp. 225-243; Zanker 1987, pp. 11-14. V. asimismo Galinsky 1999, p. 105 y Labate y Rosati 2013, p. 1. 
la historia podía insertarse más convenientemente en su obra. No obstante, los poetas más comprometidos con las ideas augusteas (Horacio y Virgilio) tendieron normalmente a una construcción en términos de interpretación, a una imagen necesaria para la comprensión de la historia, mientras que en Ovidio y Propercio, menos comprometidos, la construcción es en cierto modo un juego literario, un artificio, aunque en ocasiones también puede aportar una interpretación lateral23. Así el final del libro VI de la Eneida, que representa la futura grandeza romana, culmina en el dolor de una mors immatura, la de Marcelo, lo que constituye un significativo juicio, una medular interpretación, en términos poéticos, acerca de la historia de Roma. Como ejemplo de la segunda tendencia, Ovidio suele insertar la historia, en las Metamorfosis, conforme lo requiere la disposición de la trama, es decir supeditándola al mito y su dinámica y a la figura del poeta, con el cual la obra termina; no obstante, hay lugar para algunas interpretaciones laterales. Citemos como ejemplo precisamente el caso de Numa, cuya relación con Pitágoras y con Augusto, en Metamorfosis, se define, a través de la imagen de la pax, como una progresión que culmina en el Augusto pacificador y restaurador por medio de la guerra (XV 483 s.; XV 822 s., 833 s.); no se descuida, pues, la esfera político-militar, más allá de que la última asociación de Numa, aun no descuidando la esfera político-militar, sea, mediante los motivos de la fama y de la pax, una asociación con el poeta (XV 1 ss.; XV 483 s.; XV 853; XV 877 ss.) $)^{24}$.

En lo que atañe al presente trabajo, el lector tendería a creer que esa estructura sucesoria de Rómulo a Numa en la construcción de la imagen de Augusto, que concordaba, por lo demás, con las preferencias de Ovidio en los Fastos, puede entenderse como una interpretación del Augusto posterior a Accio, el de la pax Augusta, y a la vez como una interpretación de la historia de la Roma monárquica ${ }^{25}$; pero los hechos concomitantes y posteriores,

${ }^{23}$ Hemos analizado la construcción poética como artificio en los casos de Rómulo (Martínez Astorino 2017a), Numa (Martínez Astorino 2012) y César-Augusto (Martínez Astorino 2017b), particularmente en Metamorfosis. En lo que respecta a Propercio, pensamos en los finales de III 18 y IV 11 y, en especial, en IV, 1, IV, 4 y IV, 9, pero la lista podría incluir otras elegías. Sobre la construcción como interpretación, hemos tratado más el caso de Virgilio en su Eneida (Martínez Astorino 2014) que el de Horacio, aunque por supuesto es evidente la aplicación del concepto a este último poeta. En cuanto al episodio de Cipo en Metamorfosis, parece constituir un caso especial de interpretación, según analizamos en Martínez Astorino 2017c, pp. 268-270.

${ }^{24}$ V. Martínez Astorino 2012.

${ }^{25}$ Normalmente se está de acuerdo en que Metamorfosis y Fastos han sido compuestos entre el 2 y el 8 d.C. (en el caso de Fastos, la dedicatoria a Germánico parece corresponder a una revisión post-augustea del texto -cf. Green 2004, p. 31); sin embargo, mientras en Metamorfosis, 
las guerras externas, las auténticas dificultades de la sucesión y, en especial, la relegatio, demuestran que, con la posible excepción de la reivindicación de la monarquía de Numa, no es seguro hablar en los términos de una interpretación poética de la historia. Parece haberse tratado más bien (o en todo caso en gran medida) de un artificio del poeta, la construcción de un ilusorio mundo posible, en tensión, que debe su vida y su autenticidad a los lectores ${ }^{26}$.

\section{BIBLIOGRAFÍA}

Alföldi, A. 1973: Die zwei Loorbeerbäume des Augustus, Bonn.

Alton, E. H., Wormell, D. E. W. y Courtney, E. 2005: P. Ovidius Naso Fastorum libri sex, Múnich-Leipzig.

Austin, R. G. 1971: P. Vergili Maronis Aeneidos Liber Primus, Oxford.

Barchiesi, A. 1994: Il poeta e il principe: Ovidio e il discorso augusteo, Roma.

Barchiesi, A. 1997: The Poet and the Prince: Ovid and Augustan Discourse, Berkeley-California.

Bömer, F. 1958: P. Ovidius Naso. Die Fasten (2 tomos), Heidelberg.

por diversos motivos, uno de los cuales es la finalización en apoteosis, pero particularmente por ser una historia universal, se representa el Augusto ante Actium y el de Actium y sus consecuencias (la pacificación), el Augusto que tiene en mente el poeta de los Fastos es el Augusto post Actium, más apto para el tratamiento de los temas que se propone representar.

${ }^{26}$ A propósito de Numa y su afición a la paz, es interesante considerar aquellos versos (Aen. VI 808 ss.) en que el propio Virgilio parece deplorarla. Barchiesi 1994, p. 163 s., que retoma críticamente un comentario de Norden $1976^{6}$, p. 326 s., dice: «Anche se si dà grande importanza alla posizione di Augusto fra i due primi re di Roma, c'è da notare che quando si tratta di introdurre il terzo re della serie, Tullo Ostilio, le parole di Virgilio -cioè, ancora una volta, di Anchise, che si rivolge al figlio e lo prepara a una dura missione di guerriero- suonano come una forte riserva nei confronti del quieto Numa (Aen. 6, 812-815) ... Evidentemente il regno di Numa è risultato una pausa troppo lunga, capace di infiacchire anche se ricco di buone leggi e di religiosità. Tullo porta con sé energie e guerra, insieme con l'appropriato cliché epico arma uir-; dentro l'epos virgiliano, è un re 'epico' opposto a un re 'alieno'. Se è vero che Augusto è fiancheggiato dalla coppia Augusto-Numa, nell'ordine dei re, si misura con una coppia di antagonisti bellicosi». Ver además Monella 2008, p. 88: «In una nazione votata alla costruzione di un Impero, Numa offre il fianco all'accusa di essere un irresoluto, un imbelle; la sua 'parentesi' di non belligeranza può essere letta come una semplice perdita di tempo».

Por lo demás (y en lo que respecta a Augusto), no afirmamos, como parecería interpretar nuestro evaluador, que los Fastos representan la decepción de Ovidio acerca del Augusto que poéticamente había forjado, sino que los hechos ocurridos, si se los compara con la representación ovidiana, parecen inducir a concluir que la asociación de Augusto con Numa representada en los Fastos probablemente ha sido contrafáctica, artificial (en el sentido que hemos definido). 
Brunt, P. A. y Moore, J. M. 1973²: Res Gestae Diui Augusti. Introduction, Translation and Commentary, Oxford.

Deremetz, A. 2013: «Numa in Augustan Poetry», en Farrell, J. y Nelis, D. P. (eds.), Augustan Poetry and the Roman Republic, Oxford, pp. 228-243.

Gaffiot, F. 1934: Dictionnaire Latin-Français, París.

Galinsky, K. 1975: Ovid's Metamorphoses. An Introduction to the Basic Aspects, Oxford-Berkeley-Los Ángeles.

Galinsky, K. 1996: Augustan Culture. An Interpretive Introduction, Princeton.

Galinsky, K. 1999: «Ovid's Metamorphoses and Augustan Cultural Thematics», en Hardie, P.-Barchiesi, A.- Hinds, S. (eds.), Ovidian Transformations. Essays on the Metamorphoses and Its Reception, Cambridge, pp. 103-111.

Galinsky, K. 2013: «La costruzione del mito augusteo: Some Construction Elements», en Labate, M. y Rosati, G. (eds.), La costruzione del mito augusteo, Heidelberg, pp. 29-47.

Garani, M. 2014: «The Figure of Numa in Ovid's Fasti», en Garani, M. y Konstan, D. (eds.), The Philosophizing Muse. The Influence of Greek Philosophy on Roman Poetry, Newcastle, pp. 128-160.

Glare, P. G. W. 1968-1982: Oxford Latin Dictionary, Oxford.

Gosling, A. 2002: «Sending up the Founder: Ovid and the Apotheosis of Romulus», AClass 45, pp. 51-69.

Gowing, A. 2005: Empire and Memory. The Representation of the Roman Republic in Imperial Culture, Cambridge.

Green, S. 2004: Ovid. Fasti I. A Commentary, Brill-Leiden-Boston.

Györi, V. 2013: "Augustus and Numa: the asses of 23 BC», en Labate, M. y Rosati, G. (eds.), La costruzione del mito augusteo, Heidelberg, pp. 89-108.

Hardie, P. 2015: Ovidio. Metamorfosi, Volume VI (libri XIII-XV), Milán.

Herbert-Brown, G. 1994: Ovid and the Fasti: A Historical Study, Oxford.

Hinds, S. 1992: «Arma in Ovid's Fasti. 2. Genre. Romulean Rome and Augustan Ideology», Arethusa 25, pp. 113-153.

Labate, M. 2003: «Tra Grecia e Roma. L'identità culturale augustea nei Fasti di Ovidio», en Gazich, R. (ed.), 'Fecunda licentia'. Tradizione e innovazione in Ovidio elegiaco, Milán, pp. 71-118.

Labate, M. y Rosati, G. 2013: «Tua, Caesar, aetas: un personaggio, un'epoca, un mito. Riflessioni preliminari», en Labate, M. y Rosati, G. (eds.), La costruzione del mito augusteo, Heidelberg, pp. 1-28.

Littlewood, R. S. 2002: «Imperii pignora certa: the Role of Numa in Ovid's Fasti», en Herbert-Brown, G. (ed.), Ovid's Fasti. Historical Readings at its Bimillenium, Oxford, pp. 175-197.

Lorenzo, J. 2011: «Géneros de la memoria: retórica de la narración en la épica y la historiografía latinas», Auster 16, pp. 9-23. 
Martínez Astorino, P. 2012: «Numa y la construcción poética de la historia en las Metamorfosis de Ovidio», QUCC 102 (3), pp. 149-164.

Martínez Astorino, P. 2014: «Representaciones de la historia republicana y reciente: Marcelo en Virgilio y Propercio», Maia 66, pp. 333-351.

Martínez Astorino, P. 2017a: «Dos modos del artificio: la construcción poética de la historia en el pasaje de Rómulo de las Metamorfosis a la luz de los Fastos», Athenaeum 105, pp. 565-579.

Martínez Astorino, P. 2017b: «El poeta y la representación de la historia en Aen. 1, 223-296 у Met. 15, 761-842», AC 86, pp. 129-147.

Martínez Astorino, P. 2017c: «Cipo-César en las Metamorfosis de Ovidio: ¿una reivindicación de la monarquía?». Euphrosyne 45, pp. 259-270.

Monella, P. 2008: «L'autorità e le sue contraddizioni: Numa nei Fasti di Ovidio», en Baier, T. y Amerise, M. (eds.), Die Legitimation der Einzelherrschaft im Kontext der Generationenthematik, Berlín-Nueva York, pp. 85-108.

Norden, E. 19766: P. Vergilius Maro Aeneis Buch VI, Darmstadt.

Ogilvie, R. M. 1965: A Commentary on Livy. Books 1-5, Oxford.

Pasco-Pranger, M. 2002: «A Varronian Vatic Numa? Ovid's Fasti and Plutarch's Life of Numa», en Levene D. S. y Nelis, D. P. (eds.), Clio and the Poets: Augustan Poetry and the Traditions of Ancient Historiography, Leiden, pp. 291-311.

Ricoeur, P. 1996: «El entrecruzamiento de la historia y la ficción», en Ricoeur, P., Tiempo y narración III. El tiempo narrado, México, pp. 901-917.

Salzman, M. 1998: «Deification in the Fasti and the Metamorphoses», en Deroux, C. (ed.), Studies in Latin Literature and Roman History IX, Collection Latomus 244, Bruselas, pp. 313-346.

Stok, F. 1992: «L'alternativa dei Fasti», en Brugnoli, F. y Stok, G. (eds.), Ovidius $\pi \alpha \rho \omega \delta \eta ́ \sigma \alpha \varsigma$, Pisa, pp. 47-73.

Urban, D. 2005: Die augusteische Herrschafts-Programmatik in Ovids Metamorphosen, Fráncfort.

Wiseman, P. 1995: Remus. A Roman Myth, Cambridge.

Wiseman, P. 2002: «History, Poetry, and Annales», en Levene D. S. y Nelis, D. P. (eds.), Clio and the Poets: Augustan Poetry and the Traditions of Ancient Historiography, Leiden, pp. 331-362.

Zanker, P. 1987: Augustus und die Macht der Bilder, Múnich.

Fecha de recepción de la primera versión del artículo: 16/04/2017

Fecha de aceptación: 19/06/2017

Fecha de recepción de la versión definitiva: 12/12/2017 